\title{
Superpixel-Based Interest Points for Effective Bags of Visual Words Medical Image Retrieval
}

\author{
Sebastian Haas ${ }^{1, \star}$, René Donner ${ }^{1}$, Andreas Burner ${ }^{1}$, Markus Holzer ${ }^{1}$, \\ and Georg Langs ${ }^{1,2}$ \\ 1 Computational Image Analysis and Radiology Lab, Department of Radiology, \\ Medical University of Vienna, Austria \\ sebastian.haas@meduniwien.ac .at \\ 2 Computer Science and Artificial Intelligence Laboratory, \\ Massachusetts Institute of Technology, Cambridge, MA, USA
}

\begin{abstract}
The present work introduces a 2D medical image retrieval system which employs interest points derived from superpixels in a bags of visual words (BVW) framework. BVWs rely on stable interest points so that the local descriptors can be clustered into representative, discriminative prototypes (the visual words). We show that using the centers of mass of superpixels as interest points yields higher retrieval accuracy when compared to using Difference of Gaussians (DoG) or a dense grid of interest points. Evaluation is performed on two data sets. The ImageCLEF 2009 data set of 14.400 radiographs is used in a categorization setting and the results compare favorable to more specialized methods. The second set contains 13 thorax CTs and is used in a hybrid 2D/3D localization task, localizing the axial position of the lung through the retrieval of representative $2 \mathrm{D}$ slices.
\end{abstract}

Keywords: medical image retrieval, bags of visual words, superpixel, interest points.

\section{Introduction}

In clinical practice, radiologists rely mainly on their experience to form diagnoses. Typically the vast amount of imaging data in hospitals is not exploited due to the lack of easy to use and effective image retrieval systems. Ideally, such systems should present the radiologists with comparable cases to the one in question. In this work, we present a 2D medical image retrieval system which advances on the state of the art by incorporating a novel kind of interest points which is shown to yield very stable results on medical data.

The bags of visual words approach, for which [13] provides a good general overview, has shown promising results in medical image retrieval tasks [6]3. The main trend is the shift from purely Difference of Gaussians based interest

\footnotetext{
* The research leading to these results has received funding from the Austrian Sciences Fund (P 22578-B19, PULMARCH) and the EU-funded KHRESMOI project (FP7ICT-2009-5/257528).
} 
points to dense grids of interest points - thus effectively eliminating the influence of any interest point detector, altogether [21. The stability of the interest point detector is crucial for obtaining discriminative descriptor prototypes in the clustering stage of the bags of visual words approach. While the most popular descriptors (e.g., SIFT [8], or derivatives such as SURF [4]) are insensitive to small perturbations in the interest points, fine-grained, repetitive structures like those encountered in microscopic images [2 or lung CTs 6] prove difficult for repeatable interest point identification.

\subsection{Related Work}

The performance of several image retrieval and annotation frameworks is compared during the ImageCLEF medical tracks. Several groups have proposed methods based on BVWs in this context. TAUbiomed based a BVW dictionary on patches of 9x9 pixels in the images, and performed classification by a support vector machine (SVM) using the word histograms [3]. Dimitrovski et al. proposed a hierarchical multi-label classification, using edge histogram descriptors as global and SIFT descriptors and Local Binary Patterns (LBP) as local features [5]. Unay et al. used LBP as features together with hierarchical SVMs [10. However, none of these groups have combined the bags of visual words approach with superpixels as interest points for SIFT descriptors.

In the following Sections we outline the concept of bags of visual words and describe superpixel based interest points. In Section 3 the evaluation setup is detailed with the results presented in Section 4. We end the paper by providing a conclusion and an outlook.

\section{Methods}

We set up a flexible bags of visual words framework and replace selected parts of the BVW workflow for comparison and evaluation. In this specific case we test different interest point detectors. Section 2.1 describes the concept of the BVW framework. The three interest point detectors, we compare, are then described in Section 2.2.

\subsection{Bags of Visual Words}

BVW uses a set of training images to generate a so-called visual vocabulary derived from local image descriptors. The vocabulary is computed through clustering of all descriptors calculated from the training images. During retrieval both the training images and the query images can then be represented by a histogram of the visual words, and the distances between images correspond to distances between the histograms.

More formally, for each image $\mathbf{I}_{i}$ in the set of training images $\mathcal{I}=\mathbf{I}_{1}, \ldots, \mathbf{I}_{N}$ $K_{i}$ interest points $\mathbf{i}_{j}$ are computed. Each is comprised of coordinates $x_{j}$ and $y_{j}$, a scale $s_{j}$ and an orientation $\theta_{j}$, 


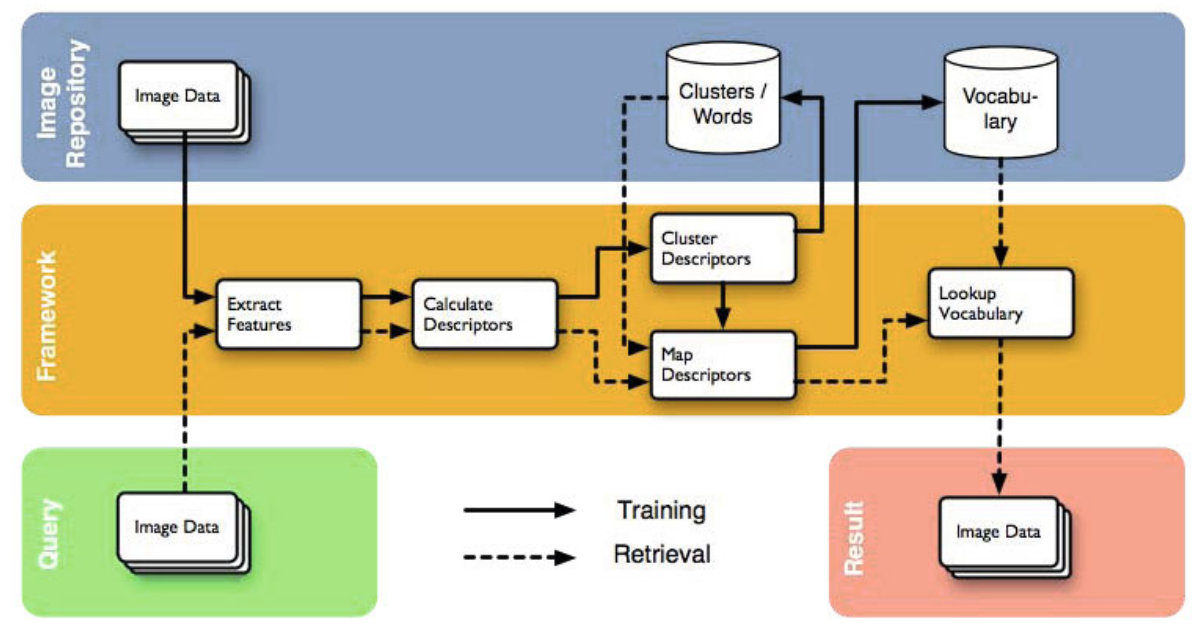

Fig. 1. Schematic overview of the Bag of Visual Words framework

$$
\mathbf{i}_{j}=\left(x_{j}, y_{j}, s_{j}, \theta_{j}\right)^{T}
$$

Corresponding with each $\mathbf{i}_{j}$ a local descriptor $\mathbf{d}_{j}$ is extracted, e. g. the $d=128$ dimensional SIFT descriptor [8]. Assembling all descriptors of all images into the $d \times D$ descriptor space $\mathbf{D}$ allows to perform clustering. Typically $k$-means clustering is used. The resulting $k d$-dimensional cluster centers $\mathbf{c}_{l}$ represent the visual words.

To obtain the histogram $\mathbf{h}_{i}$ for image $\mathbf{I}_{i}$ we perform vector quantization on all its descriptors $\mathbf{d}_{j}$ onto the cluster centers $\mathbf{c}_{l}$ : Each $\mathbf{d}_{j}$ is assigned the label $l$ of the closest cluster, using Euclidean distance in the descriptor space. Forming a histogram over all the labels encountered within an image yields the $k$-bin histogram $\mathbf{h}_{i}$, which is normalized to sum to 1 .

Retrieval The retrieval step is similar to the processing of one training image. The interest points and corresponding descriptors are extracted for the query image $\mathbf{I}_{q}$ and mapped to the visual words. The histogram $\mathbf{h}_{q}$ based on the cluster mapping is used in a nearest neighbor query using the $\chi^{2}-$ distance against the database of previous stored histograms.

Figure 1 shows the training and retrieval pipeline used in the BVW framework.

\subsection{Interest Points: DoG, DENSE, Superpixel Points}

We evaluate the influence of varying the type of interest points on the quality of the BVW retrieval result. We compare SIFT interest points (DoG keypoints), DENSE points (regular grid) and superpixel points. 


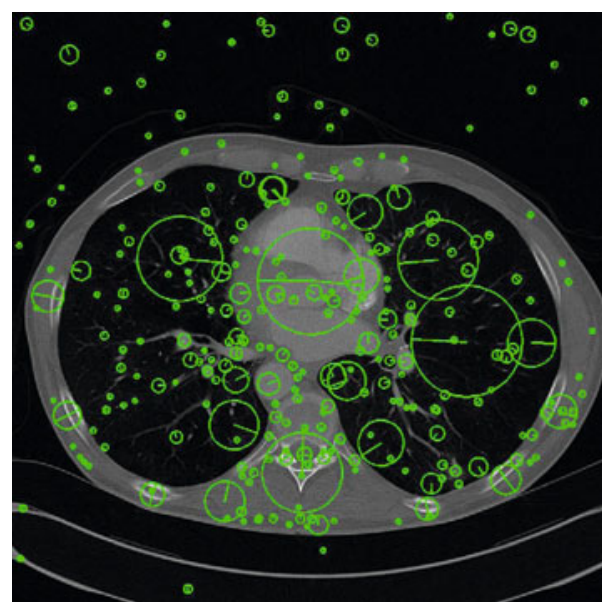

(a)

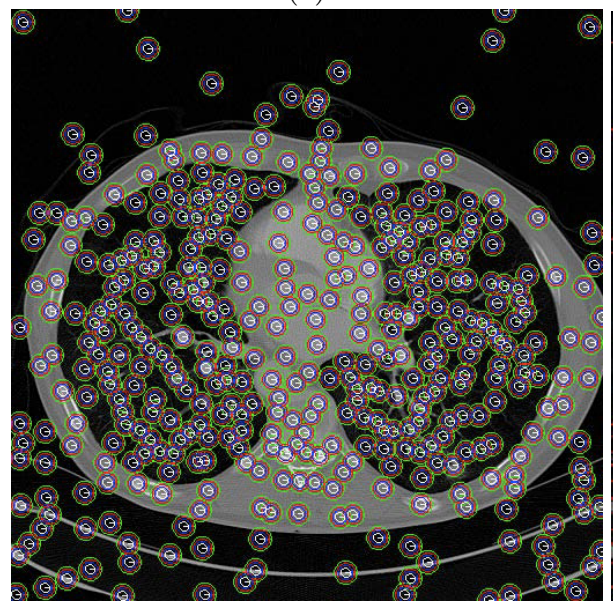

(c)

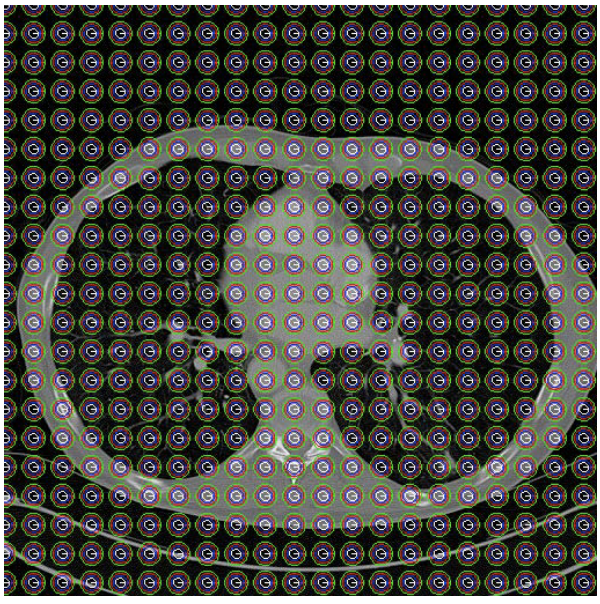

(b)

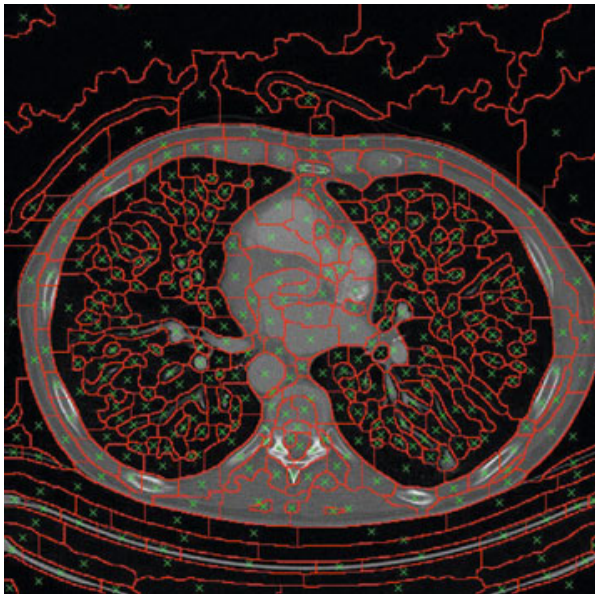

(d)

Fig. 2. An example lung image showing interest point position, orientation and scale for a) SIFT b) DENSE c) superpixel d) shows the the superpixel regions as well as the centers of mass used as interest points

SIFT: The Scale-Invariant Features introduced by Lowe are widely used in the field of image processing. Being invariant against scaling, rotation or translation of the image helps finding similar image regions in manipulated or even different images. We use the standard implementation provided by 11 to perform our experiments. An example for SIFT interest points showing their location, scale and orientation can be found in Figure 2 (a).

DENSE: Dense SIFT as used in [2] is based on a regular grid over the image. The interest points used for the computation of the SIFT descriptors are generated 
based on the size of the image. The content of the image does not affect the DENSE interest points. The interest points are located on a regular grid relative to the image coordinate system. Constant values are used for the orientation and scale needed for the computation of SIFT descriptors. In our setup we assign each point an orientation of $0^{\circ}$ and scales of 4, 6, 8 and 10 pixels leading to four interest points per location. In contrast to Andreé [2], our interest points at not overlapping. We use a rather wide grid spacing of 25 pixels to reduce the amount of interest points to a similar value like SIFT or superpixels compute. The dense grid of interest points is shown in Figure 2 (b).

Superpixel points: Superpixels represent an over-segmentation of the underlying image into partitions which exhibit maximal internal homogeneity and maximal inter-super pixel heterogeneity, while respecting image boundaries. Several different approaches for conforming with this loose definition have been proposed. They vary in terms of smoothness, regularity and computational cost. In this paper, a superpixel implementation following [12] was used due to the low computational cost and the low number of resulting superpixels in background areas. The chosen method employs the monogenic signal to compute the local phase. This measure is invariant to changes in contrast and brightness. Local phase means that each pixel contains directional information to the strongest edge within a radius $r$ corresponding to the selected scale of the monogenic signal. This property makes the monogenic signal very well suited for medical image data, with their often subtle edge gradient which defy conventional edge detectors. A watershed transform on the monogenic signal yields the regions which are used as superpixels in our approach.

To obtain interest points from these regions the center of mass of each region is computed. Again, the orientation is set to $0^{\circ}$ and four interest points with a scales of 4,6,8 and 10 pixels are created per center.

Figure 2 (c) shows the superpixel interest points for an example lung slice. In (d) the borders and centers of mass are shown for all superpixel regions.

\subsection{Distribution of Interest Points in the Image}

Figure 3 shows an example slice of a lung CT (a) and the distribution of SIFT (b) and superpixel (c) interest points on this image. The distribution of the interest points is shown as a heat map image with 25 by 25 bins. Each bin represents an area of approximately 20 by 20 pixels of the original image. The heat map for the DENSE interest points was left out because of their uniform distribution.

The superpixel heat map shows a lower density in the upper part of the image. This area shows mainly the air above the thorax. The superpixel algorithm generates in this area fewer, but bigger superpixels. Compared to SIFT the superpixel algorithm generates in the area of the lung more uniform distributed interest points. 


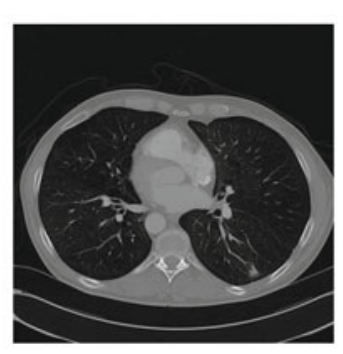

(a)

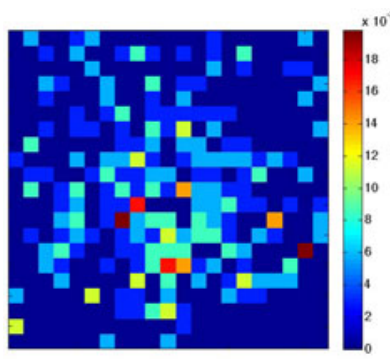

(b)

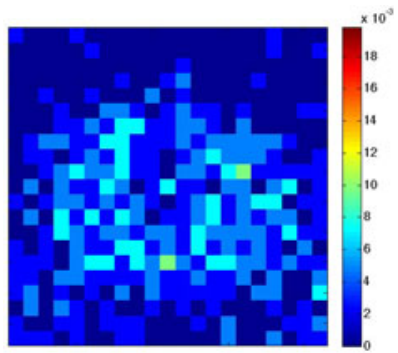

(c)

Fig. 3. Based on the image shown in a) the distribution of the SIFT and superpixel interest points are shown in b) and c), respectively. The number of interest points per type are normalized to obtain comparable heat maps. Note the more even and more concentrated distribution in the area of the medical object of interest.

\section{Experiments}

In this section we describe two independent experiments for testing the three types of interest points.

The first experiment uses the bags of visual words framework for an annotation task. This task is based on the ImageCLEF 2009 Medical Image Annotation Task 9. The used image repository 11 contains $14.410 \mathrm{x}$-ray images split into a training set (12.677 images) and a test set (1.733 images). All images are fully annotated with the IRMA code [7.

The IRMA code for each test set image is derived from the retrieval result image with the highest similarity. We assign each query image the IRMA code corresponds to the closest result image in the training data. The classified images were evaluated with the scheme 2 used by the ImageCLEF 2009 Medical Image Annotation Task. Although this scheme allows wildcards within the IRMA code, we don't use them. Our experiment only takes the classification set 2008 into account, which is the most complete classification and based on the IRMA code. The other three sets 2005, 2006 (both are sets of simple raw numbers) and 2007 (IRMA code) are not used.

The second experiment uses the BVW framework for locating CT slices of lung CTs based on the position of the retrieved images. Histograms of visual words in combination with dense SURF descriptors have been successfully used for estimating body portions using CT volumes [6]. For this run we use 13 lung CTs, each CT containing between 132 and 530 slices with a resolution of $512 \times 512$ pixel. The slices of each CT containing parts of the lung are registered on a scale from 0.0 to 1.0 where the proximal occurrence of the lung in the volume has the value 0.0 and the distal occurrence has the value 1.0. These two key-images

\footnotetext{
1 http://www . irma-project . org/datasets . php?SELECTED=00009\#00009. dataset

2 http://www.idiap.ch/clef2009/evaluation_tools/error_evaluation.pdf
} 
are selected manually. All slices in between get equally distributed coordinates assigned. The slices cranial of the first lung-slice get negative values and the slices caudad of the last lung-slice get values greater than 1.0. Figure 4 shows an ordered subset of images taken from one CT with their assigned position value. The first and last occurrence of the lung are marked. The 13 lung CTs are processed with the BVW framework using a leave-one-out cross-validation. From every CT 100 images with equally spaced positions between 0.0 and 1.0 are selected as query images. Based on this query images we test the location accuracy for the slices of the whole lung CT. The position is determined by the median position value of the 10 most similar retrieved images per query.

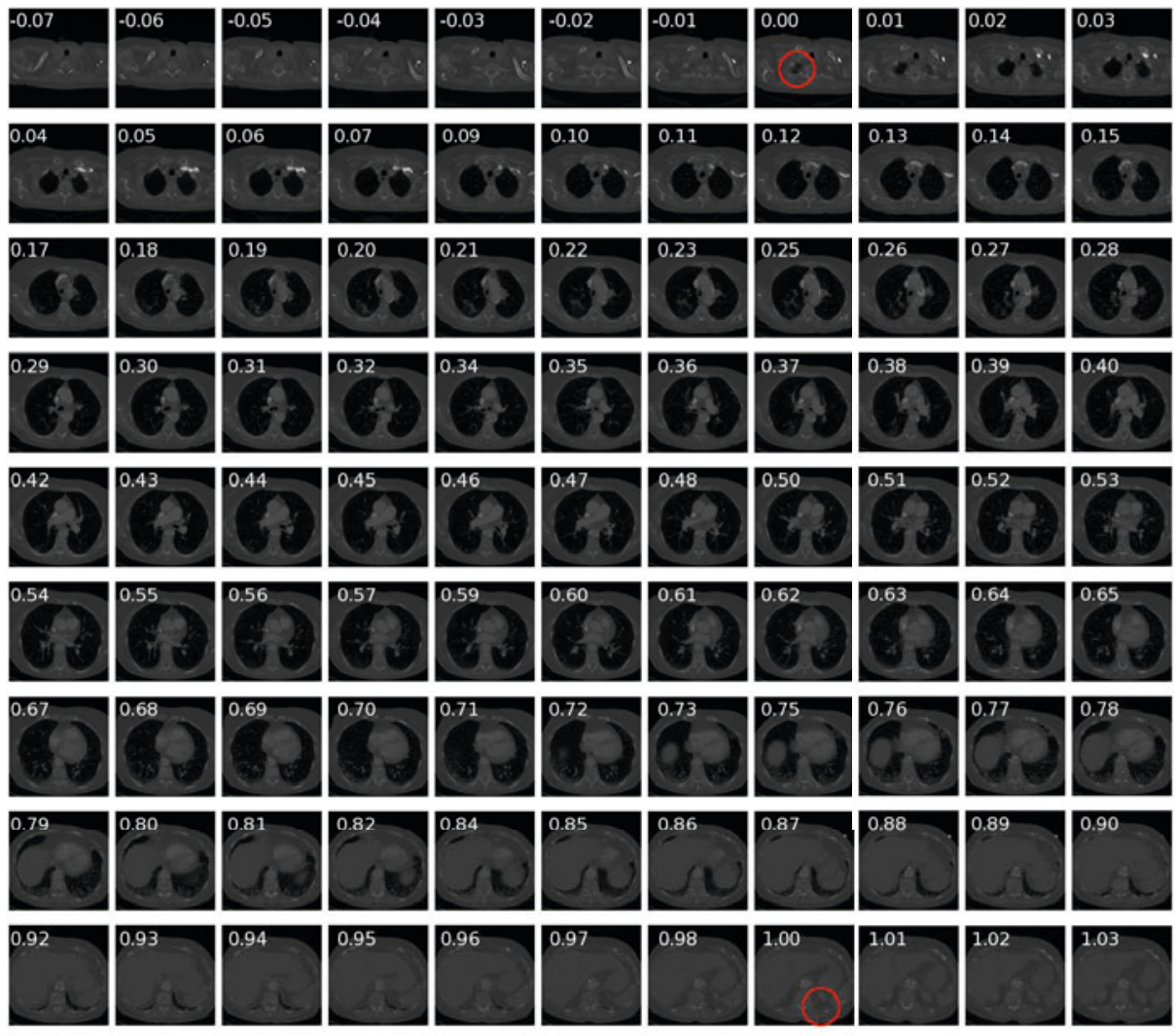

Fig. 4. Slices from one example CT with first and last occurrence of the lung marked. The numbers indicate the registered positions of the slices.

Both experiments are set up for SIFT, DENSE and superpixel interest points. The BOW dictionary is build from 1000 images leading to approximately $1.6 * 10^{6}$ to $2.0 * 10^{6}$ interest points and descriptors. These descriptors are clustered to 1000 words using k-means. 


\section{Results}

The error scores of the first experiments are calculated by using the scoring schema defined for the ImageCLEF 2009 Medical Image Annotation Task. The superpixel approach clearly outperforms the approaches with DENSE and SIFT interest points with a score of 249.34 against 442.51 and 562.75 , respectively. Table 1 shows the best run of each competitor of the ImageCLEF in addition to the three approaches evaluated in this paper.

Although the superpixel approach shows a good performance, we expect a better values by improving the selection of IRMA code by e. g. taking more than the first retrieved image into account.

Table 1. This table shows the error score of the three approaches described in this paper (bold) and the best result of each group from the ImageCLEF 2009 Medical Image Annotation Task 9]. The error score is based on the evaluation scheme for the IRMA code classification set 2008. A low score indicates a higher accuracy.

\begin{tabular}{lr}
\hline Approach & error score \\
\hline TAUbiomed & 169.50 \\
Idiap & 178.93 \\
FEITIJS & 242.46 \\
Superpixels & $\mathbf{2 4 9 . 3 4}$ \\
VPA SabanciUniv & 261.16 \\
MedGIFT & 317.53 \\
IRMA & 359.29 \\
DENSE & $\mathbf{4 4 2 . 5 1}$ \\
SIFT & $\mathbf{5 6 2 . 7 5}$ \\
DEU & 642.50 \\
\hline
\end{tabular}

For the second experiment, for each leave-one-out run, we retrieve the 10 most similar images for each of the the 100 images from test CT. The position is determined by the median position value of the ten best retrieved images per query. Knowing the position of each query image in the ground truth, we are able to aggregate the computed image-positions over all $13 \mathrm{CTs}$. The 13 leave one out runs are aggregated by computing the mean position for each of the 100 test slices. Figure 5 shows plots of the mean value and standard deviation of the aggregated data for the SIFT, DENSE and superpixel interest points. The x-axis of the plots defines the image position in the ground truth whilst the mean value and the standard deviation is plotted against the y-axis. The baseline shown as the $45^{\circ}$ line indicates the optimal result. The plots show that the mean values of the superpixel image-positions are closer to the baseline than in the results of the other two runs.

The overall performance of the three interest point types is summed up on Table 2. Superpixels with a sum-of-squared-erros of 0.0554 perform better than SIFT and DENSE interest points with 0.1416 and 0.1453 respectively. 


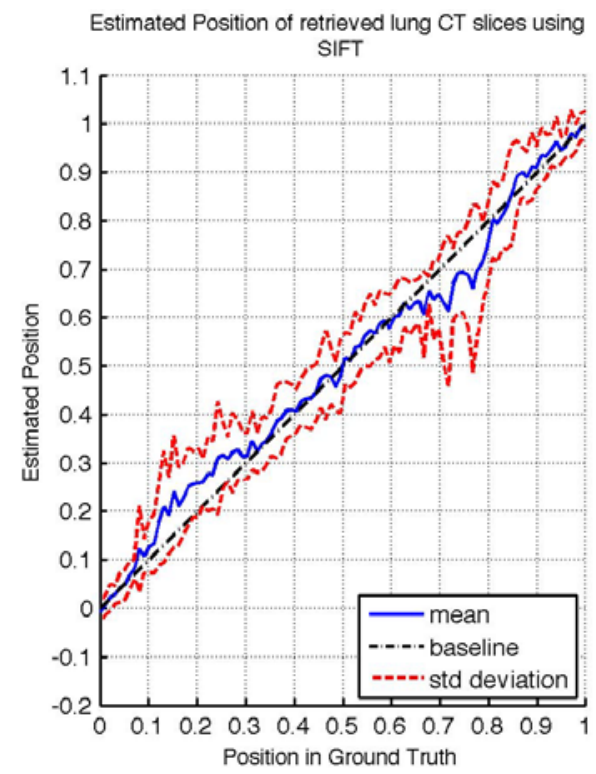

(a)

Estimated Position of retrieved lung CT slices using SuperPixelPoints

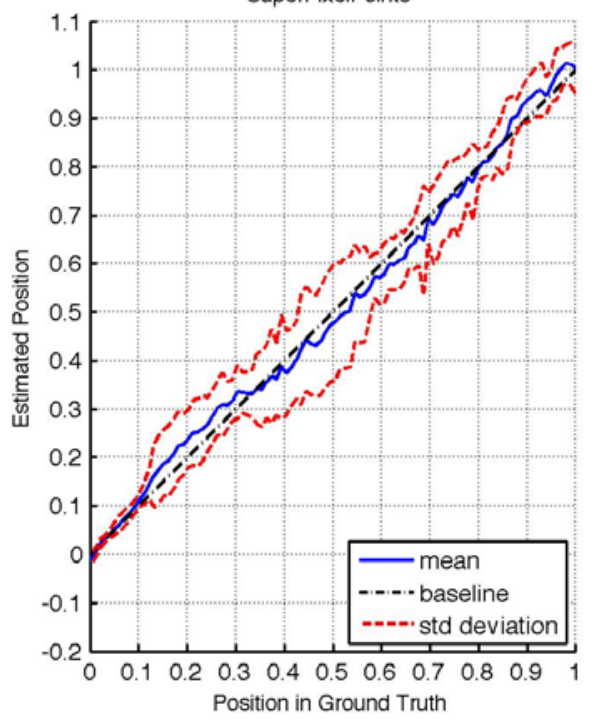

Estimated Position of retrieved lung CT slices using DENSE

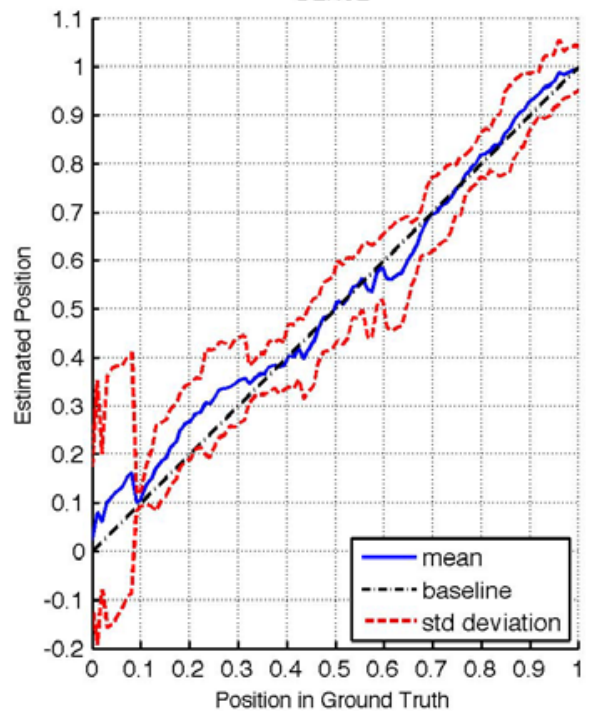

(b)

(c)

Fig. 5. Results of location retrieval using (a) SIFT, (b) DENSE and (c) superpixel interest points. The known position of the 100 query images are plotted on the $\mathrm{x}$-axis. The y-axis shows the computed image position. The plots show the optimal result (baseline), the mean position and standard deviation for each query position aggregated over the 13 CTs. 
Table 2. Aggregated deviation of our test results shown in Figure 5. Mean, median and sum of squared errors (SSE) for the distance between our results and the baseline of the three lung CT position estimation runs.

\begin{tabular}{cclc}
\hline Interest points & mean & median & SSE \\
\hline SIFT & 0.0278 & $\mathbf{0 . 0 1 8 6}$ & 0.1416 \\
DENSE & 0.0295 & 0.0233 & 0.1453 \\
Superpixel & $\mathbf{0 . 0 1 9 9}$ & 0.0234 & $\mathbf{0 . 0 5 5 4}$ \\
\hline
\end{tabular}

\section{Conclusion}

In this paper we have introduced and evaluated a medical image retrieval and localization system which extends the Bags of Visual Words approach to using interest points derived from superpixels. Superpixels provide a stable oversegmentation of image structures, and their centers of mass thus yield stable interest points. This is particularly relevant for medical imaging data, where both salient points, and homogeneous structures occur at unique locations across a population. This in turn allows the clustering stage in BVWs to find discriminative prototypes which improve the retrieval accuracy of the proposed system.

For both tasks, the classical image category classification and the $2 \mathrm{D} / 3 \mathrm{D}$ localization task, superpixel interest points yielded better results, while being straightforward and fast to compute. Future work will evaluate different types of superpixels for their stability and extend the proposed retrieval system to $3 \mathrm{D}$ data.

\section{References}

1. André, B., Vercauteren, T., Perchant, A., Wallace, M.B., Buchner, A.M., Ayache, N.: Endomicroscopic image retrieval and classification using invariant visual features. In: Proceedings of the Sixth IEEE International Symposium on Biomedical Imaging 2009 (ISBI 2009), pp. 346-349. IEEE, Boston (2009)

2. André, B., Vercauteren, T., Wallace, M.B., Buchner, A.M., Ayache, N.: Endomicroscopic video retrieval using mosaicing and visual words. In: Proceedings of the Seventh IEEE International Symposium on Biomedical Imaging 2010 (ISBI 2010), pp. 1419-1422. IEEE (2010)

3. Avni, U., Goldberger, J., Greenspan, H.: Addressing the ImageCLEF 2009 challenge using a patch-based visual words representation. In: Working Notes for the CLEF 2009 Workshop. The Cross-Language Evaluation Forum (CLEF), Corfu, Greece (2009)

4. Bay, H., Tuytelaars, T., Van Gool, L.: SURF: Speeded Up Robust Features. In: Leonardis, A., Bischof, H., Pinz, A. (eds.) ECCV 2006. LNCS, vol. 3951, pp. 404-417. Springer, Heidelberg (2006)

5. Dimitrovski, I., Kocev, D., Loskovska, S., Džeroski, S.: ImageCLEF 2009 Medical Image Annotation Task: PCTs for Hierarchical Multi-Label Classification. In: Peters, C., Caputo, B., Gonzalo, J., Jones, G.J.F., Kalpathy-Cramer, J., Müller, H., Tsikrika, T. (eds.) CLEF 2009. LNCS, vol. 6242, pp. 231-238. Springer, Heidelberg (2010) 
6. Feulner, J., Zhou, S.K., Seifert, S., Cavallaro, A., Hornegger, J., Comaniciu, D.: Estimating the body portion of CT volumes by matching histograms of visual words. In: Medical Imaging 2009: Image Processing (Proceedings Volume), vol. 7259, p. 72591. SPIE (2009)

7. Lehmann, T.M., Schubert, H., Keysers, D., Kohnen, M., Wein, B.B.: The IRMA code for unique classification of medical images. In: Medical Imaging 2003: PACS and Integrated Medical Information Systems: Design and Evaluation (Proceedings Volume), vol. 5033, pp. 440-451. SPIE (2003)

8. Lowe, D.G.: Distinctive image features from scale-invariant keypoints. International Journal of Computer Vision 60, 91-110 (2004)

9. Tommasi, T., Caputo, B., Welter, P., Güld, M.O., Deserno, T.M.: Overview of the CLEF 2009 Medical Image Annotation Track. In: Peters, C., Caputo, B., Gonzalo, J., Jones, G.J.F., Kalpathy-Cramer, J., Müller, H., Tsikrika, T. (eds.) CLEF 2009. LNCS, vol. 6242, pp. 85-93. Springer, Heidelberg (2010)

10. Ünay, D., Soldea, O., Akyüz, S., Çetin, M., Erçil, A.: Medical image retrieval and automatic annotation: VPA-SABANCI at ImageCLEF 2009. In: Working Notes for the CLEF 2009 Workshop. The Cross-Language Evaluation Forum (CLEF), Corfu, Greece (2009)

11. Vedaldi, A., Fulkerson, B.: Vlfeat: an open and portable library of computer vision algorithms. In: Proceedings of the International Conference on Multimedia, MM 2010, pp. 1469-1472. ACM, New York (2010)

12. Wildenauer, H., Mičušík, B., Vincze, M.: Efficient Texture Representation using Multi-Scale Regions. In: Yagi, Y., Kang, S.B., Kweon, I.S., Zha, H. (eds.) ACCV 2007, Part I. LNCS, vol. 4843, pp. 65-74. Springer, Heidelberg (2007)

13. Zhang, J., Marszalek, M., Lazebnik, S., Schmid, C.: Local features and kernels for classification of texture and object categories: A comprehensive study. In: Conference on Computer Vision and Pattern Recognition Workshop, p. 13 (June 2006) 